\title{
LUMINOSITY LOSS RATE \\ DUE \\ TO INTRABEAM SCATTERING
}

A. G. RUGGIERO

(BNL, December 11, 1983) 


$$
\text { RHIC-PG-21 }
$$

Luminosity Loss Rate due to Intrabeam Scattering

A.g. Ruggieno

BNL, Decenfer 11, 1983 
(1)

In the limit of short interaction region luminosity can be very well represented by

the

$$
L=\frac{N^{2} B f_{\text {rev }}}{4 \pi \sigma_{v}^{*} \sigma_{H}^{*} \cdot f}
$$

where, for identical colliding bears,

$N$, number of parties per bunch

$B$, number of bunches ger Jean

tres, revolution frequency

$\sigma_{v}^{*}$, vertical rms beam ride at the collision point

$\sigma_{H}^{*}$, horizontal rms beam size at the collision joint

assuming crossing at an angle on the hon: octal plane

$$
f=\sqrt{1+p^{2}}
$$

and

$$
p=\frac{\alpha \sigma_{e}}{2 \sigma_{H}^{*}}
$$


(2)

$\alpha$, total crossing angle $\sigma_{e}$, rms bunch length.

Intrabeam scattering has the effect of Laving $\sigma_{v}^{*}, \sigma_{H}^{*}$ and $\sigma_{E}$, the r.m.s energy spread to vary, generally by growing.

Define the luminosity loss rate

$$
\tau_{L}^{-1}=-\frac{1}{L} \frac{d L}{d t}
$$

Obviously:

$$
\tau_{L}^{-1}=\frac{1}{\sigma_{v}^{*}} \frac{d \sigma_{v}^{*}}{d t}+\frac{1}{\sigma_{H}^{*}} \frac{d \sigma_{H}^{*}}{d t}+\frac{1}{f} \frac{d f}{d t}
$$

One can work out that

$$
\frac{1}{f} \frac{d f}{d t}=\frac{p^{2}}{f^{2}}\left[\frac{1}{\sigma_{l}} \frac{d \hat{\sigma}_{l}}{d t}-\frac{1}{\sigma_{H}^{*}} \frac{d \hat{\sigma}_{H}^{*}}{d t}\right]
$$


(3)

Assumptions:

1. The bunch length increases (or decreases) at the same rate the energy spread does, that is

$$
\frac{1}{\sigma_{e}} \frac{d \sigma_{e}}{d t}=\tau_{e}^{-1}
$$

2. We assume the at the two modes of oscillations (H and V) are Lully coyle to each stolen on a time shorter than the intratean scat? taring diffusion times, that is

$$
\frac{1}{\sigma_{v}^{*}} \frac{d \sigma_{v}^{*}}{d t}=\frac{1}{\sigma_{H}^{*}} \frac{d \sigma_{H}^{*}}{d t}=\tau_{H}^{-1}+\tau_{v}^{-1}
$$

Here $\tau_{E}^{-1}, \tau_{H}^{-1}$ and $\tau_{\nu}^{-1}$ are the diffusion (or damping rates) from intrabeam scattering respectively in energy, horizontal (betatron) size and vertical (betatron) size. 
(4)

In conchnsion

$$
\tau_{L}^{-1}=\frac{p^{2}}{f^{2}} \tau_{E}^{-1}+\left(2-\frac{p^{2}}{f^{2}}\right)\left(\tau_{H}^{-1}+\tau_{v}^{-1}\right)
$$

For head-on collision $p=0, f=1$ and

$$
\tau_{l}^{-1}=2\left(\tau_{H}^{-1}+\tau_{\nu}^{-1}\right)
$$

